\title{
Wavelength conversion in a highly nonlinear chalcogenide microstructured fiber
}

\author{
Sy Dat Le, ${ }^{1,2, *}$ Mathilde Gay, ${ }^{1,2}$ Laurent Bramerie,,${ }^{1,2}$ Marcia Costa e Silva, ${ }^{1,2}$ Kevin Lenglé, ${ }^{1,2}$ \\ Thierry Chartier, ${ }^{1,2}$ Monique Thual, ${ }^{1,2}$ Jean-Claude Simon, ${ }^{1,2}$ Laurent Brilland, ${ }^{3}$ David Méchin, ${ }^{3}$ \\ Perrine Toupin, ${ }^{1,4}$ and Johann Troles, ${ }^{1,4}$ \\ ${ }^{1}$ Université europenne de Bretagne, 5 Boulevard Lannec, 35000 Rennes, France \\ ${ }^{2}$ CNRS Foton (UMR 6082), 6 rue de Kerampont, BP 80518, 22305 Lannion cedex, France \\ ${ }^{3}$ PERFOS, RED platform of Photonics Bretagne, 11 rue Louis de Broglie, 22300 Lannion, France \\ ${ }^{4}$ CNRS Sciences Chimiques de Rennes (UMR 6226), Université de Rennes 1, 35000 Rennes, France \\ ${ }^{*}$ Corresponding author: sy-dat.le@enssat.fr \\ Compiled May 23, 2013

\begin{abstract}
We report on all-optical wavelength conversion of a $56 \mathrm{~Gb} / \mathrm{s}$ differential quadrature phase shift keying signal and a $42.7 \mathrm{~Gb} / \mathrm{s}$ on-off keying signal. Wavelength conversion is based on four-wave mixing effect in a $1 \mathrm{~m}$-long highly nonlinear GeAsSe chalcogenide fiber. The high nonlinearity of the fiber allows low power penalty operation with a total average power of less than $60 \mathrm{~mW}$. (C) 2013 Optical Society of America

OCIS codes: $060.4005,190.4380,070.4340$.
\end{abstract}

All-optical signal processing, such as amplification, regeneration, time-domain demultiplexing or wavelength conversion, has recently drawn a lot of interests. Among these all-optical functions, wavelength conversion is expected to play a useful role in the future optical network to achieve wavelength flexibility. Wavelength conversion can be performed by semiconductor optical amplifiers, saturable absorbers or other nonlinear materials. The advantage of nonlinear materials with strong Kerr effect is their fast response-time and their passive operation. This make them excellent candidates for high-bit-rate all-optical signal processing in general and wavelength conversion in particular.

Due to their high Kerr nonlinearity, chalcogenide glasses are one of the most favorite choices to fabricate highly-nonlinear components. The nonlinear refractive index of chalcogenide glasses may be greater than that of fused silica several hundred times. Recently, a chalcogenide fiber with a suspended-core structure has been reported [1]. Its core diameter, as small as $1.13 \mu \mathrm{m}$, has allowed reaching a nonlinear coefficient up to $46000 \mathrm{~W}^{-1} \mathrm{~km}^{-1}$. However, this fiber exhibited a multimode behavior.

By utilizing nonlinear components made of chalcogenide glasses, some studies have been carried out to perform all-optical wavelength conversion based on fourwave mixing (FWM). The first demonstration has been proposed by V. G. Ta'eed et al. at $10 \mathrm{~Gb} / \mathrm{s}$ in a $1 \mathrm{~m}$-long AsSe chalcogenide fiber [2] with a peak power of $2.1 \mathrm{~W}$. L. B. Fu et al. reported a $40 \mathrm{~Gb} / \mathrm{s}$ wavelength conversion in a tapered chalcogenide fiber with a total average power of $180 \mathrm{~mW}$ [3]. Recently, M. D. Pelusi et al. have successfully demonstrated wavelength conversion of a $40 \mathrm{~Gb} / \mathrm{s}$ differential phase shift keying (DPSK) signal in a AsS chalcogenide waveguide with a total average power of $400 \mathrm{~mW}$ [4].
In this letter, we present a $1 \mathrm{~m}$-long chalcogenide fiber with a single-mode behavior. Its Kerr nonlinearity of about $11000 \mathrm{~W}^{-1} \mathrm{~km}^{-1}$ allows error-free FWM-based wavelength conversion with a total average power of less than $60 \mathrm{~mW}$. The fiber is first briefly presented. Experimental results on wavelength conversion of a $42.7 \mathrm{~Gb} / \mathrm{s}$ on-off keying (OOK) signal and a $56 \mathrm{~Gb} / \mathrm{s}$ DQPSK signal will be presented.

The fiber is fabricated according to the casting method described in Ref. [5]. The glass composition is $\mathrm{Ge}_{10} \mathrm{As}_{22} \mathrm{Se}_{68}$. To fabricate the fiber, a microstructured preform is prepared with a structure of three rings of holes around a solid core. The GeAsSe chalcogenide microstructured fiber is then drawn from the preform by applying differential pressures in the holes. The nonlinear refractive index $n_{2}$ of the GeAsSe glass is estimated between 400 times and 500 times greater than that of fused silica.

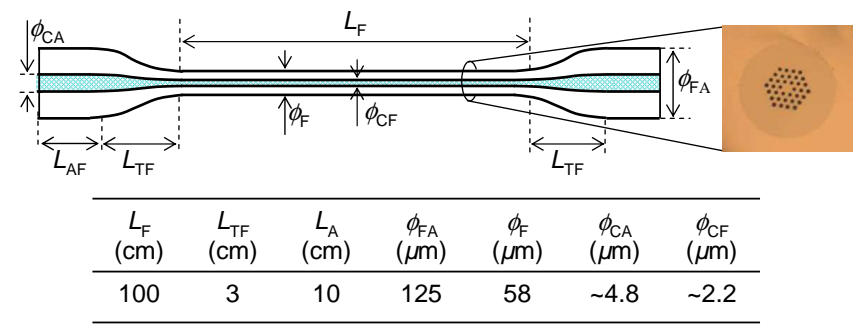

Fig. 1. Chalcogenide GeAsSe microstructured fiber.

In order to reach a high nonlinear coefficient $\gamma$, the fiber has been drawn with a core diameter as small as possible. However, small-core fibers are difficult to couple with conventional fibers. For this reason, our fiber is drawn with different core diameters as shown in Fig. 1. The input and output ends of the fiber are drawn with a core diameter $\phi_{C A}$ of $4.75 \mu \mathrm{m}$, while the main part of 
the fiber is drawn with a core diameter $\phi_{C F}$ of $2.2 \mu \mathrm{m}$. Thanks to this tapering process during the drawing, the fiber offers the advantage of a highly-nonlinear part (the waist) and mode-adaptation parts at two ends to facilitate the coupling. The length $L_{F}$ of the nonlinear part of the fiber is $1 \mathrm{~m}$. The length $L_{A}$ of the mode-adaptation parts is $10 \mathrm{~cm}$, and the length $L_{T F}$ of the tapers is around $3 \mathrm{~cm}$. The total length of the fiber is $1.26 \mathrm{~m}$.

The coupling in the microstructured fiber is realized by using a Gradhyp microlensed fiber [6] with a mode field diameter of $4.8 \mu \mathrm{m}$, very close to the input and output core diameters of the fiber. This allows us to limit the coupling loss. The ratio between the power at the output of the microlensed fiber and the power at the output of the chalcogenide fiber is $6.6 \mathrm{~dB}$ at $1550 \mathrm{~nm}$. This value includes the loss due to Fresnel reflections $(1 \mathrm{~dB}$ per facet for this glass) and the attenuation of the fiber which is $2.5 \mathrm{~dB} / \mathrm{m}$ in our case. The additional losses of $1.4 \mathrm{~dB}$ are due to coupling loss and taper loss.

Based on self-phase modulation (SPM), the nonlinear coefficient $\gamma$ and the dispersion $D$ of the fiber have been estimated. A mode-locked laser emitting Gaussian pulses of $8 \mathrm{ps}$ at $1550 \mathrm{~nm}$ with a repetition rate of $20 \mathrm{MHz}$ was used for SPM characterization. By comparing the experimental and the simulated spectral broadening, we obtained a nonlinear coefficient $\gamma$ of $11000 \mathrm{~W}^{-1} \mathrm{~km}^{-1}$ and a group-velocity dispersion $D$ of $-500 \mathrm{ps} / \mathrm{km} / \mathrm{nm}$ [7].

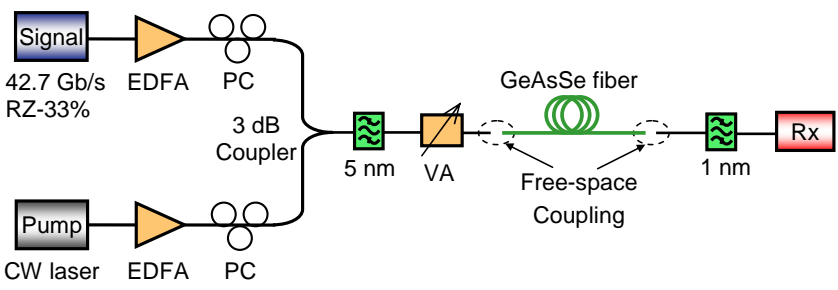

Fig. 2. Experimental setup for wavelength conversion.

Utilizing the GeAsSe fiber, we implemented the FWMbased wavelength conversion, in which the new wave at a new wavelength is generated from a signal wave and a pump wave. The experimental setup for wavelength conversion of $42.7 \mathrm{~Gb} / \mathrm{s}$ signal is depicted in Fig. 2. The $42.7 \mathrm{~Gb} / \mathrm{s}$ return-to-zero (RZ) transmitter generates pulses at $1553 \mathrm{~nm}$ with a full-width at half-maximum of $8.3 \mathrm{ps}$. The $42.7 \mathrm{~Gb} / \mathrm{s}$ signal is amplified by an erbium doped fiber amplifier (EDFA). The tunable continuous wave (CW) pump is amplified by a second EDFA. After amplifiers, both signal and CW waves pass through polarization controllers (PC) and are combined by a 3-dB coupler. Both waves are filtered by an optical flat-top band-pass filter of $5 \mathrm{~nm}$ to reject the amplified spontaneous emission noise of EDFAs. To adjust the power launched into the chalcogenide fiber, a variable attenuator (VA) is used just after the $5 \mathrm{~nm}$ filter. The optical power is coupled into and out of the fiber using the microlensed fibers as described previously.

The CW pump wavelength is tuned to set the wavelength of the converted signal. Fig. 3a illustrates an ex- ample of output spectrum for a wavelength detuning $\Delta \lambda$ of $2.6 \mathrm{~nm}$ with respect to the data signal. The spectrum clearly exhibits first-order and second-order idler waves. In this case, the total average power at the output of the microlensed fiber is $56 \mathrm{~mW}$ with a CW power of $26 \mathrm{~mW}$. By taking into account injection loss around $2.4 \mathrm{~dB}$, we estimate that the total average power launched into the nonlinear part of the GeAsSe fiber is about $32 \mathrm{~mW}$.
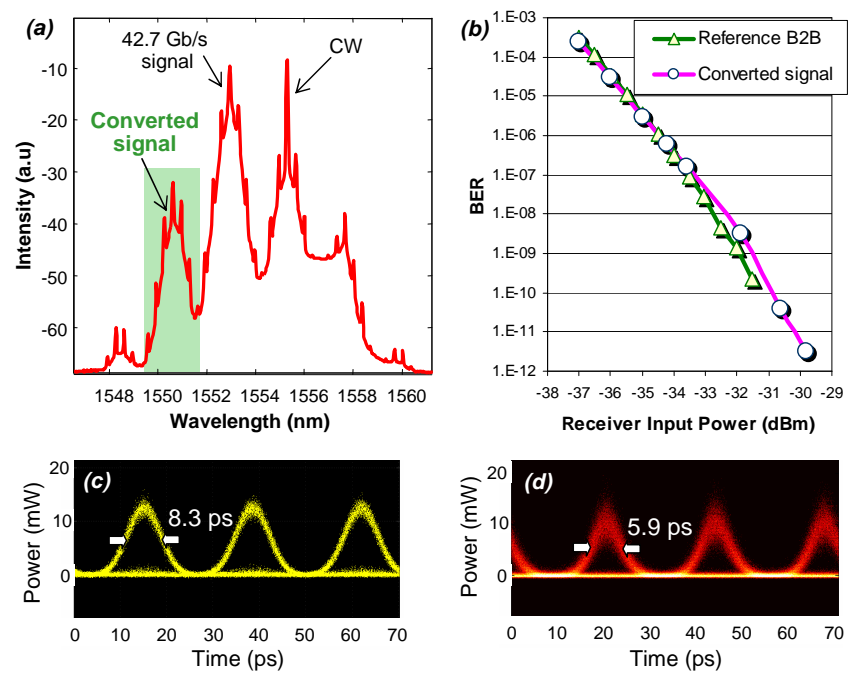

Fig. 3. (a) Spectrum at the output of GeAsSe fiber, (b) BER measurement, and eye-diagram of (c) original and (d) converted $42.7 \mathrm{~Gb} / \mathrm{s}$ signal.

To assess the FWM conversion efficiency of the firstorder idler wave, we have measured the ratio between the collected peak power of the idler wave at the output of the chalcogenide fiber and the signal power at $42.7 \mathrm{~Gb} / \mathrm{s}$ injected in the chalcogenide fiber. The FWM conversion efficiency is found to be $-27 \mathrm{~dB}$ with a pump power launched into the chalcogenide fiber of only $15 \mathrm{~mW}$ and a detuning $\Delta \lambda$ of $2.6 \mathrm{~nm}$.

To analyse the converted signal, a $1 \mathrm{~nm}$ band-pass filter centered at $1550.4 \mathrm{~nm}$ is used after the GeAsSe fiber. The filtered signal is amplified by an EDFA before detection. Performance of the wavelength conversion is assessed by bit-error-rate (BER) measurements versus receiver input power for a $42.7 \mathrm{~Gb} / \mathrm{s}$ pseudorandom bit sequence (PRBS) length of $2^{31}-1$ bits. The Fig. $3 \mathrm{~b}$ shows the evolution of the BER for the back-to-back (B2B) case and for the converted signal. Error free wavelength conversion is achieved. A negligible power penalty of $0.4 \mathrm{~dB}$ is found for a BER of $10^{-9}$.

Fig. 3c and 3d show the eye-diagrams of the original and converted $42.7 \mathrm{~Gb} / \mathrm{s}$ signals, respectively. Note in Fig. 3d that the optical signal-to-noise ratio is much lower than in Fig. 3c due to less available power for the $42.7 \mathrm{~Gb} / \mathrm{s}$ converted signal. The pulse duration of the converted signal is measured to be $5.9 \mathrm{ps}$. It agrees with the theory that the converted pulses are compressed by a factor of the square root of 2 from the original pulse of duration $8.3 \mathrm{ps}$. This is due to the fact that the idler 
signal is proportional, in our case, to the square of the original $42.7 \mathrm{~Gb} / \mathrm{s}$ signal.

In order to determine the conversion range, we have measured FWM efficiency as a function of the wavelength detuning $\Delta \lambda$. The results are presented in Figure 4. They have been obtained with two CW pumps with a total power of $15.3 \mathrm{dBm}$. From this curve, we estimate that the conversion range at $3 \mathrm{~dB}$ is about $2.8 \mathrm{~nm}$.

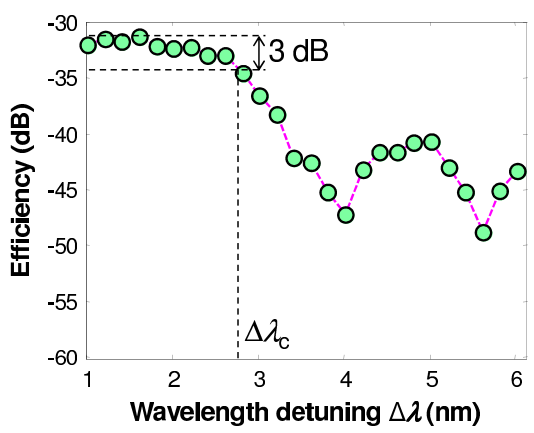

Fig. 4. FWM efficiency versus wavelength detuning

We now consider the $56 \mathrm{~Gb} / \mathrm{s}$ DQPSK wavelength conversion. The DQPSK signal is generated by a nested Mach-Zehnder modulator with $2^{15}-1$ bit length PRBS. The experimental setup for $56 \mathrm{~Gb} / \mathrm{s}$ DQPSK wavelength conversion is the same as for the $42.7 \mathrm{~Gb} / \mathrm{s}$ OOK signal. The wavelength of the DQPSK signal is centered at $1556.2 \mathrm{~nm}$. The wavelength of the converted signal is changed with the CW pump wavelength tuning. In the experiment, the wavelength of the CW pump is set to $1557.8 \mathrm{~nm}$ leading to a detuning $\Delta \lambda$ of $1.6 \mathrm{~nm}$ between the signal and the pump. Since the DQPSK spectrum is narrower than the OOK spectrum, we choose a smaller detuning in order to have more FWM efficiency.

An example of optical spectrum at the output of the GeAsSe fiber is shown in Fig. 5a. It exhibits FWM waves shifted by $\Delta \lambda$ on both sides of the initial waves. Contrary to OOK wavelength conversion, DQPSK wavelength conversion should preserve the phase of the signal. The converted signal must then be the one proportional to the input signal, not to the square of the input signal. In our experiment, it corresponds to the wave close to the CW pump, centered at $1559.4 \mathrm{~nm}$. At the output of the GeAsSe fiber, a $1 \mathrm{~nm}$ band-pass filter centered at $1559.4 \mathrm{~nm}$ is used to extract the converted signal. The total average power at the output of the VA is set to $45 \mathrm{~mW}$, including a CW pump power of $25 \mathrm{~mW}$. The power of the converted signal after the $1 \mathrm{~nm}$ filter is measured to be $-22 \mathrm{dBm}$.

The receiver uses a fiber-based delay-line interferometer and balanced detectors for BER analysis. Fig. 5b shows BER measurements of the converted signal as a function of the power on the receiver. The B2B case is also represented for comparison. A power penalty of about $1 \mathrm{~dB}$ for a BER of $10^{-9}$ is found. Error-free wavelength conversion of a $56 \mathrm{~Gb} / \mathrm{s}$ DQPSK signal has therefore been demonstrated with a conversion shift of $3.2 \mathrm{~nm}$
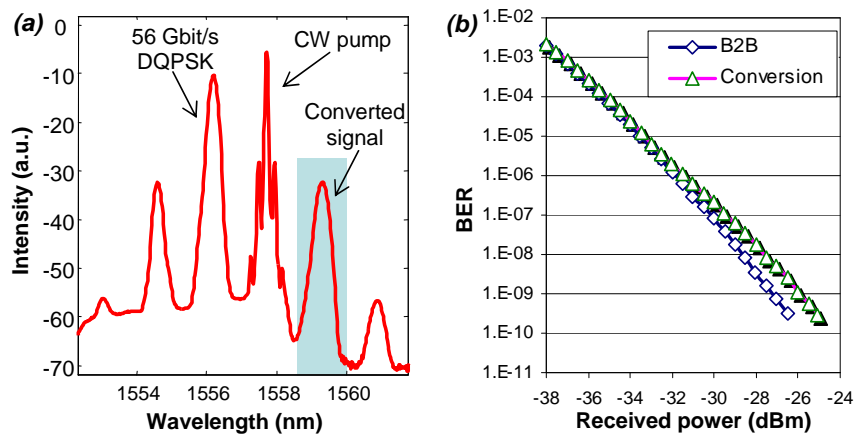

Fig. 5. (a) Spectrum at the output of GeAsSe fiber, and (b) $56 \mathrm{~Gb} / \mathrm{s}$ DQPSK BER measurement.

(twice the detuning $\Delta \lambda$ ) and a total average power of $45 \mathrm{~mW}$. Due to the strong value of the fiber dispersion, the detuning $\Delta \lambda$ is limited to about $3 \mathrm{~nm}$ and may not allow to process signals with bit rates higher than $80 \mathrm{~Gb} / \mathrm{s}$.

To conclude, a new chalcogenide GeAsSe microstructured fiber with a nonlinear coefficient of $11000 \mathrm{~W}^{-1} \mathrm{~km}^{-1}$ and attenuation loss of $2.5 \mathrm{~dB} / \mathrm{m}$ is presented. We have experimentally demonstrated, for the first time to our best knowledge, a $56 \mathrm{~Gb} / \mathrm{s}$ DQPSK and a $42.7 \mathrm{~Gb} / \mathrm{s}$ OOK FWM-based wavelength conversion in a microsctructured chalcogenide fiber. Error-free wavelength conversion has been obtained for both cases with total average powers lower than $60 \mathrm{~mW}$. These results show the capability of chalcogenide microstructured fibers into all-optical signal processing at high-bit-rate.

This work is supported by the Conseil Régional de Bretagne, the Conseil Général des Côtes d'Armor, the European Union Feder program and the French government.

\section{References}

1. S. D. Le, D. M. Nguyen, M. Thual, L. Bramerie, M. Costa e Silva, K. Lenglé, M. Gay, T. Chartier, L. Brilland, D. Méchin, P. Toupin, and J. Troles, Optics Express 19, B653B660 (2011).

2. V. Ta'Eed, L. Fu, M. Pelusi, M. Rochette, I. Littler, D. Moss, and B. Eggleton, Optics Express 14, 10371 (2006).

3. L. Fu, M. Pelusi, E. Magi, V. Ta'eed, and B. Eggleton, Electronics Letters 44, 44 (2008).

4. M. Pelusi, F. Luan, S. Madden, D. Choi, D. Bulla, B. Luther-Davies, and B. Eggleton, Photonics Technology Letters, IEEE 22, 3 (2010).

5. Q. Coulombier, L. Brilland, P. Houizot, T. Chartier, T. Nguyen, F. Smektala, G. Renversez, A. Monteville, D. Méchin, T. Pain, H. Orain, J.-C. Sangleboeuf, and J. Troles, Optics Express 18, 9107 (2010).

6. M. Thual, P. Rochard, P. Chanclou, and L. Quetel, Fiber and Integrated Optics 27, 532 (2008).

7. S. D. Le, D. Nguyen, M. Thual, L. Bramerie, M. Costa e Silva, K. Lenglé, M. Gay, T. Chartier, J.-C. Simon, L. Brilland, D. Méchin, P. Toupin, and J. Troles, in OFC Conference (Optical Society of America, 2012). 


\section{Informational Fourth Page}

In this section, please provide full versions of citations to assist reviewers and editors (OL publishes a short form of citations) or any other information that would aid the peer-review process.

\section{References}

1. S. D. Le, D. M. Nguyen, M. Thual, L. Bramerie, M. Costa e Silva, K. Lengle, M. Gay, T. Chartier, L. Brilland, D. Mechin, P. Toupin, and J. Troles, "Efficient four-wave mixing in a ultra-highly nonlinear suspendedcore chalcogenide $\mathrm{As}_{38} \mathrm{Se}_{62}$ fiber", Optics Express 19, B653B660 (2011).

2. V. Ta'Eed, L. Fu, M. Pelusi, M. Rochette, I. Littler, D. Moss, and B. Eggleton, "Error free all optical wavelength conversion in highly nonlinear AsSe chalcogenide glass fiber", Optics Express 14, 10371 (2006).

3. L. Fu, M. Pelusi, E. Magi, V. Ta'eed, and B. Eggleton, "Broadband all-optical wavelength conversion of 40 Gbit/s signals in nonlinearity enhanced tapered chalcogenide fibre", Electronics Letters 44, 44 (2008).

4. M. Pelusi, F. Luan, S. Madden, D. Choi, D. Bulla, B. Luther-Davies, and B. Eggleton, "Wavelength conversion of high-speed phase and intensity modulated signals using a highly nonlinear Chalcogenide glass chip", Photonics Technology Letters, IEEE 22, 3 (2010).

5. Q. Coulombier, L. Brilland, P. Houizot, T. Chartier, T. NGuyen, F. Smektala, G. Renversez, A. Monteville, D. Méchin, T. Pain, H. Orain, J.-C. Sangleboeuf, and J. Troles, "Casting method for producing lowloss chalcogenide micro-structured optical fibers", Optics Express 18, 9107 (2010).

6. M. Thual, P. Rochard, P. Chanclou, and L. Quetel, "Contribution to research on Micro-Lensed Fibers for Modes Coupling", Fiber and Integrated Optics 27, 532 (2008).

7. S. D. Le, D. Nguyen, M. Thual, L. Bramerie, M. Costa e Silva, K. Lenglé, M. Gay, T. Chartier, J.-C. Simon, L. Brilland, D. Méchin, P. Toupin, and J. Troles, " 42.7 Gbit/s RZ-33\% wavelength conversion in a Chalcogenide microstructured fiber", in Optical Fiber Communication Conference (Optical Society of America, 2012). 